\title{
Convective heat transfer in a closed two-phase thermosyphon
}

\author{
M.A. Al-Ani ${ }^{1,2,3, a}$ \\ ${ }^{1}$ Institute of Power Engineering, National Research Tomsk Polytechnic University, Tomsk, Russia \\ ${ }^{2}$ Mechanical engineering Department College of engineering, the University of Mustansiriyah, \\ Baghdad, Iraq \\ ${ }^{3}$ Faculty of Mechanics and Mathematics, Tomsk State University, Tomsk, Russia
}

\begin{abstract}
A numerical analysis of heat transfer processes and hydrodynamics in a twophase closed thermosyphon in a fairly wide range of variation of governing parameters has been investigated. A mathematical model is formulated based on the laws of mass conservation, momentum and energy in dimensionless variables "stream function - vorticity vector velocity - temperature". The analysis of the modes of forced and mixed convection for different values of Reynolds number and heat flows in the evaporation zone, the possibility of using two-phase thermosyphon for cooling gas turbine blades, when the heat is coming from the turbine blades to the thermosyphon is recycled a secondary refrigerant has been studied with different values of the centrifugal velocity. Nusselet Number, streamlines, velocity, temperature fields and temperature profile has been calculated during the investigation.
\end{abstract}

\section{Introduction}

One of the main problems in the design of power equipment of thermal power plants operating at high pressures and temperatures is to ensure optimum thermal conditions of such equipment. Thus, used cooling systems (e.g., air, gas turbine blades) result in decrease in gas turbines efficiency in general.

The solution to this problem is possible by using a sufficiently broad set of systems and thermal mode control devices. One of such devices, which is called two-phase thermosyphon, and is used to cool turbine blades, has been studied quite intensively in the 60 years of the last century. But so far these devices are not widely used in industry in general and in power systems, in particular, though numerous experimental investigations have been carried out, confirming high (or even very high) heat transfer ability of thermosyphons. This is largely due the fact that there is no theory of heat transfer in such devices, describing the whole complexity of interrelated energy transfer processes in the vapor channel, a membrane of liquid coolant, flowing on the walls, and in the cylinder case, which is the main structural component of thermosyphon. Separate solutions of specific problems met in statements, not

\footnotetext{
${ }^{a}$ Corresponding author: maathe_a@yahoo.com
}

This is an Open Access article distributed under the terms of the Creative Commons Attribution License 4.0, which permits unrestricted use, distribution, and reproduction in any medium, provided the original work is properly cited. 


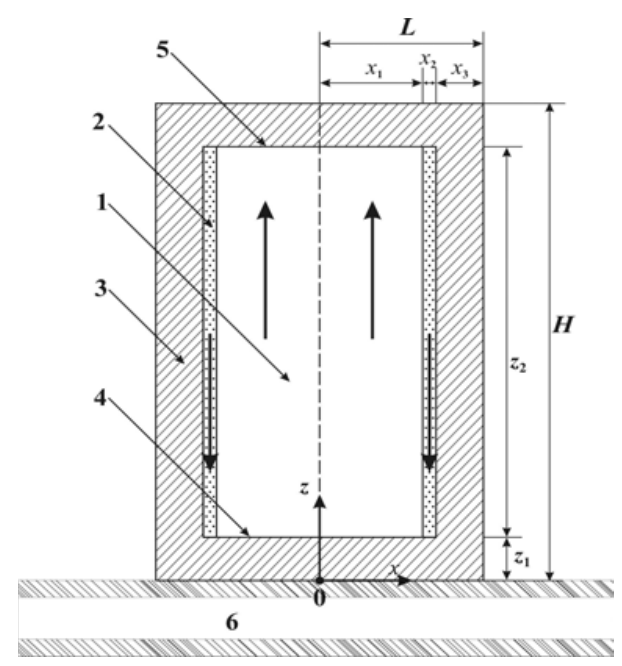

Figure 1. Schematic diagram of the thermosyphon, 1 - vapor, 2 - liquid membrane, 3 - metal shell, 4 - surface evaporation, 5 - condensation surface, 6 - circuit of power equipment cooler element movement.

taking into account conjugation - the corresponding influence of heat transfer processes in vapor and liquid phases, as well as in the casing [1].

To justify the possibility of using thermosyphons at the regulation of thermal modes of power equipment individual elements in thermal power plants it is necessary to construct a theory based on sufficient empirical evidences explaining the heat and mass transfer in the thermosyphon as a system consisting of a number of its interacting components: the source of energy supply, the zone of evaporation and condensation, the steam channel, the membrane of condensate falling under gravity, the constructing thermosyphon heat transfer, and mechanical components of the casing. Experimental investigations on a level that provides reliable prediction of thermosyphons efficiency in conditions appropriate to power equipment operation modes in thermal power plants, is very time consuming, costly and does not guarantee an optimal result. Complex set of interrelated processes in the thermosyphon is impossible to analyze analytically. The only real method of the problem investigation is the numerical simulation. To date, there were no known results of numerical studies of the basic heat and mass transfer laws in thermosyphons, applied to the operating conditions of systems for ensuring thermal mode of thermal power stations main equipment.

The aim of the thesis is mathematical modeling of fluid flow and heat transfer in a closed, two-phase thermosyphon, which is one of the most promising elements of power equipment cooling systems in thermal power plants - gas turbine blades.

Analysis of the literature on the problem under investigation shows that despite the variety of possible uses of thermosyphons two main configurations can be considered basic to any application, which are thermosyphon in the form of cylinders and rectangular cross-section. It may be noticed that the latter configuration, in many cases is preferable for the opportunity to ensure good contact with the cooling zone in a large area without any extra transitional devices. At the same time the cylindrical thermosyphon can be used in certain cooling systems, although for the power systems the most attractive thermosyphons are rectangular cross-sections. In connection with the foregoing, the dissertation examines both configurations. In the formulation of the problem, the maximum discretization of research on areas of basic physical processes (vapor channel, membrane condensation, the wall thermosyphon, the zone of evaporation and condensation, the interface "vapor-liquid, the liquidwall"). 


\section{Mathematical model}

The mathematical formulation of the thesis solved the basic problem of convective heat transfer in dimensionless variables in Cartesian-cylindrical coordinate system in the form:

$$
\begin{aligned}
& \frac{\partial \Omega_{1}}{\partial \tau}+\frac{1}{X^{\kappa}}\left(\frac{\partial \Psi_{1}}{\partial Z} \frac{\partial \Omega_{1}}{\partial X}-\frac{\partial \Psi_{1}}{\partial X} \frac{\partial \Omega_{1}}{\partial Z}\right)=\vartheta_{1}^{\mathrm{nc}}\left[\frac{1}{X^{\kappa}} \frac{\partial}{\partial X}\left(X^{\kappa} \frac{\partial \Omega_{1}}{\partial X}\right)+\frac{\partial^{2} \Omega_{1}}{\partial Z^{2}}\right] \\
& +\kappa \frac{\Omega_{1}}{X^{2}}\left(\frac{\partial \Psi_{1}}{\partial Z}-\vartheta_{1}^{\mathrm{nc}}\right)+\vartheta_{\mathrm{fc}} \frac{\partial \Theta_{1}}{\partial X} \\
& \frac{\partial^{2} \Psi_{1}}{\partial X^{2}}+\frac{\partial^{2} \Psi_{1}}{\partial Z^{2}}-\frac{\kappa}{X} \frac{\partial \Psi_{1}}{\partial X}=-X^{\kappa} \Omega_{1} \\
& \frac{\partial \Theta_{1}}{\partial \tau}+\frac{1}{X^{\kappa}}\left(\frac{\partial \Psi_{1}}{\partial Z} \frac{\partial \Theta_{1}}{\partial X}-\frac{\partial \Psi_{1}}{\partial X} \frac{\partial \Theta_{1}}{\partial Z}\right)=\frac{\vartheta_{1}^{\mathrm{nc}}}{\operatorname{Pr}_{1}}\left[\frac{1}{X^{\kappa}} \frac{\partial}{\partial X}\left(X^{\kappa} \frac{\partial \Theta_{1}}{\partial X}\right)+\frac{\partial^{2} \Theta_{1}}{\partial Z^{2}}\right] \\
& V_{2}=\frac{\varphi-\rho_{2} g_{y}}{(2+2 \kappa) \mu_{2} V_{0}} z_{2}^{2}\left(X_{1}+X_{2}-X\right)+C_{1}\left[(1-\kappa)\left(X_{1}+X_{2}-X\right)+\kappa \ln \left(X_{1}+X_{2}-X\right)\right]+C_{2} ; \\
& \Psi_{2}=\int_{X_{1}}^{X_{1}+X_{2}} V_{2} d X \\
& \frac{\partial \Theta_{2}}{\partial \tau}=\frac{\vartheta_{2}^{\mathrm{nc}}}{\operatorname{Pr}_{2}}\left[\frac{1}{X^{\kappa}} \frac{\partial}{\partial X}\left(X^{\kappa} \frac{\partial \Theta_{2}}{\partial X}\right)+\frac{\partial^{2} \Theta_{2}}{\partial Z^{2}}\right] ;
\end{aligned}
$$

for the thermosyphon casing:

$$
\frac{\partial \Theta_{3}}{\partial \mathrm{Fo}_{\mathrm{O}}}=\frac{1}{X^{\kappa}} \frac{\partial}{\partial X}\left(X^{\kappa} \frac{\partial \Theta_{3}}{\partial X}\right)+\frac{\partial^{2} \Theta_{3}}{\partial Z^{2}} ;
$$

Where $X, Z$ - transverse (radial) and longitudinal (axial) axis of a cartesian (cylindrical) coordinate system, for a flat course - for the ax-symmetric flow:

$$
\begin{aligned}
\vartheta_{i}^{\mathrm{nc}} & =\left\{\begin{array}{l}
\sqrt{\frac{\mathrm{Pr}_{i}}{\mathrm{Ra}_{i}}}-\text { in the case of natural convection, } \\
\frac{1}{\mathrm{Re}_{i}}-\text { in the case of mixed or forced convection, }
\end{array} \quad i=1,2 ;\right.
\end{aligned}
$$

$\operatorname{Pr}=\frac{v}{a}-$ Prandtl number, $v-$ kinematic viscosity; a - thermal diffusivity, Fo $=\frac{a_{3} t_{0}}{z_{2}^{2}}-$ Fourier number, $\mathrm{Ra}=\frac{g_{y} \beta\left(T_{h}-T_{c}\right) z_{2}^{3}}{\nu a}-$ the Rayleigh number, $\tau-$ the dimensionless time; $\mathrm{V}_{0}-$ the scale of the velocity (speed convection); $\Theta$ - dimensionless temperature; $\Psi$ - dimensionless stream function, $\Omega$ - dimensionless analog of the curl, $\operatorname{Re}_{1}=W_{\text {исп }} z_{2} / v$ - Reynolds number, $W_{\text {Исп }}$ - the rate of liquid evaporation. Constants $\mathrm{C} 1$ and $\mathrm{C} 2$ are determined from boundary conditions. Analysis of heat transfer intensity on the surface of condensation and evaporation was conducted by the values $\overline{N u}=$ $\int_{0}^{X_{1}}\left|\frac{\partial \Theta_{1}}{\partial Z}\right| d X$ of average Nusselt number. 


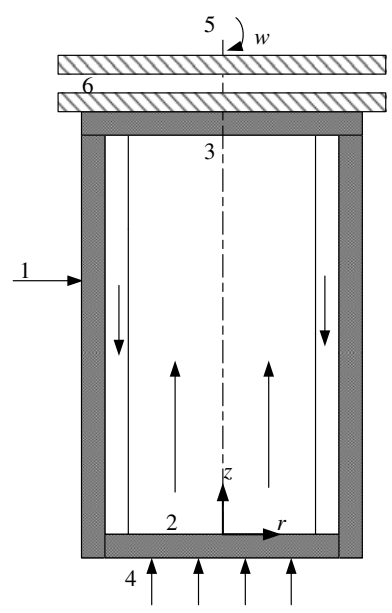

Figure 2. Thermosyphon for cooling gas turbine blades, 1 - thermosyphon case, 2 - evaporation zone, 3 condensation zone, 4 - gas heat flow, 5 - rotor, 6 - duct with refrigerant.

The initial conditions for system (1) - (6):

$$
\Psi(X, Z, 0)=\Omega(X, Z, 0)=\Theta(X, Z, 0)=0 .
$$

Boundary conditions:

$$
\begin{aligned}
& X=0, \frac{z_{1}}{z_{2}} \leq Z \leq \frac{z_{1}+z_{2}}{z_{2}}, \frac{\partial \Theta}{\partial X}=0, \Psi=0 \\
& X=\frac{L}{z_{2}}, \quad 0 \leq Z \leq \frac{H}{z_{2}}, \frac{\partial \Theta}{\partial X}=0
\end{aligned}
$$

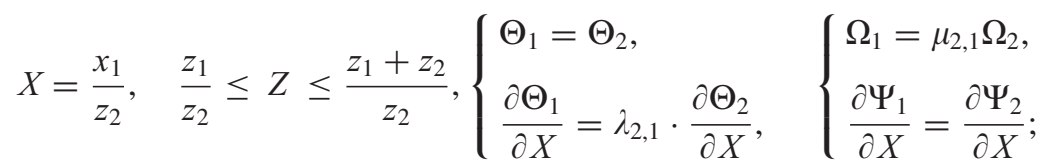

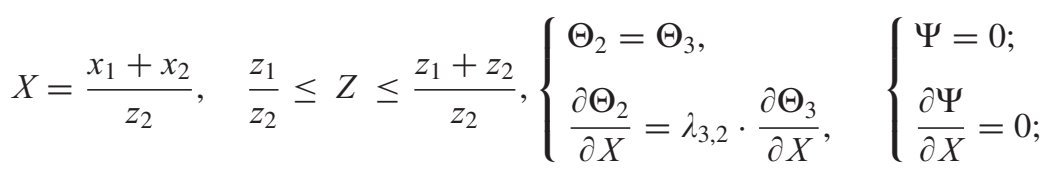

$$
\begin{aligned}
& Z=0, \quad 0 \leq X \leq \frac{L}{z_{2}}, \Theta=\Theta_{h} \\
& Z=\frac{H}{z_{2}}, \quad 0 \leq X \leq \frac{L}{z_{2}}, \frac{\partial \Theta}{\partial Z}=\operatorname{Bi}\left(\Theta_{\mathrm{e}}-\Theta\right) \\
& Z=z_{1} / z_{2}, \quad 0 \leq X \leq \frac{x_{1}}{z_{2}},-\frac{\partial \Theta_{3}}{\partial Z}=-\lambda_{1,3} \cdot \frac{\partial \Theta_{1}}{\partial Z}-\breve{Q}_{u c n} W_{u c n}, \Theta_{3}=\Theta_{1} ; \\
& Z=z_{1}+z_{2} / z_{2}, \quad 0 \leq X \leq \frac{x_{1}}{z_{2}},-\frac{\partial \Theta_{1}}{\partial Z}=-\lambda_{3,1} \cdot \frac{\partial \Theta_{3}}{\partial Z}+\breve{Q}_{K O H} W_{K O H}, \Theta_{1}=\Theta_{3} .
\end{aligned}
$$



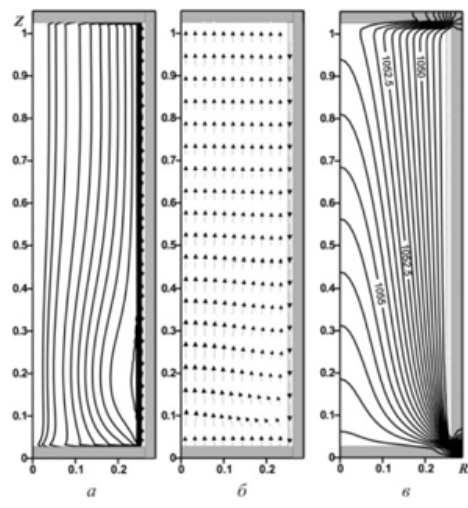

Figure 3. Streamlines (a) velocity field (b) and temperature (c) at a $=25 \mathrm{~g}$.

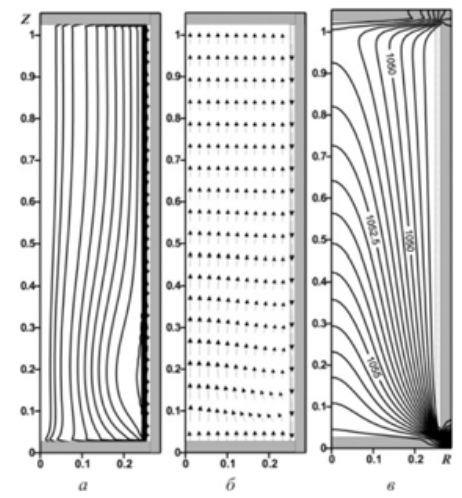

Figure 4. Streamlines (a) velocity field (b) and temperature (c) at $\mathrm{a}=50 \mathrm{~g}$.

where $\lambda_{2,1}=\lambda_{2} / \lambda_{1}$ - relative thermal conductivity coefficient, $\mu_{2,1}=\mu_{2} / \mu_{1}-$ the relative dynamic viscosity, and; $\breve{Q}_{\text {исп }}, \breve{Q}_{\text {Кон }}$ and $W_{\text {исп }}, W_{\mathrm{KOH}}$ - the dimensionless heat and the rate of evaporation and condensation, $\mathrm{Bi}=\frac{\alpha z_{2}}{\lambda_{3}}-$ Biot number, $\Theta \mathrm{e}-$ dimensionless temperature of the environment.

\section{Results}

The formulated problem with corresponding initial and boundary conditions have been solved by finite differences method on a uniform grid using an implicit two-layer scheme. To approximate convective terms, monotone approximation of A.A. Samarsky of second-order accuracy, taking into account peculiarities of the analyzed process, for diffusion terms - symmetric differences. Parabolic equations have been solved on the basis of local one-dimensional scheme of A.A. Samarsky. The approximation of the equation for the stream function (1) has been carried out using five-point template "cross". To solve the algebraic equations resulting system the method of Gauss-Seidel have been used, which acceleration was achieved using the method of upper relaxation. The optimal relaxation parameter value has been chosen on the basis of numerical experiments [5].

Figures 3-6 show the results of numerical investigations of heat transfer in a two-phase closed thermosyphon. The analysis of investigated process thermohydrodynamic features at heat fluxes in the 


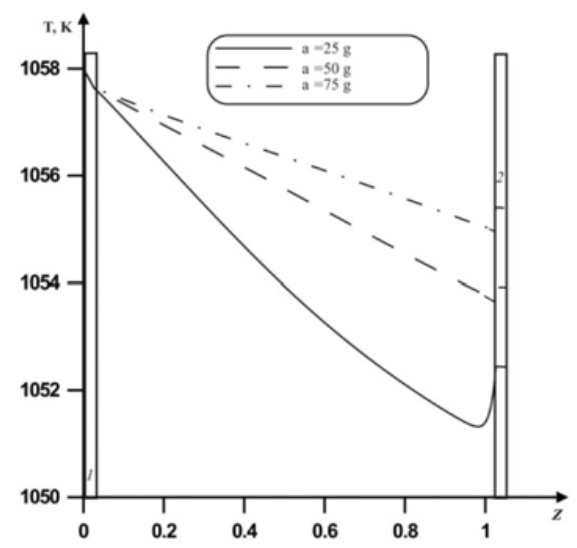

Figure 5. Temperature profiles in the cross section $\mathrm{R}=0$.

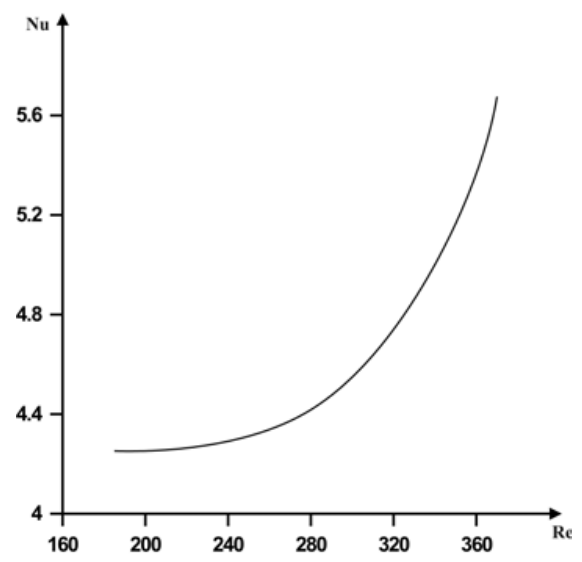

Figure 6. Dependence of average Nusselt number on Re.

evaporation zone, corresponding to thermal power equipment conditions. At the problem statement, an option of thermosyphon (which is a structural component of a gas turbine blade) rotation with a high speed was considered. Under these conditions, the condensate is returned to the evaporation zone (high temperatures area) by centrifugal forces, due to thermosyphon rotation, and one of the main process parameters is the centripetal acceleration affecting condensate membrane. To the condensation zone steam moves through steam path under the influence of forces due to the pressure drop.

Numerical investigations have been carried out for cylindrical thermosyphons with steel walls. As a working special refrigerants based on alkali metals have been considered. Typical geometric characteristics of thermosyphon have been chosen: height $-100 \mathrm{~mm}$, an internal specific transverse size $-25 \mathrm{~mm}$, wall thickness $-2.5 \mathrm{~mm}$.

Analysis of mixed convection modes at different values of heat flow into the evaporation zone has been carried out.

The possibility of using two-phase thermosyphon for cooling gas turbine blades, when the heat, coming from the turbine blades to thermosyphon, is recycled by a secondary refrigerant (Fig. 2).

Figures 3 and 4 show typical streamlines, velocity and temperature areas for different value of acceleration (rotating velocity) at a temperature in the evaporation zone thermosyphon of $780^{\circ} \mathrm{C}$. 
The distribution of $\mathrm{T}(\mathrm{Z})$ in Fig. 5 shows the extent of the influence of acceleration on the temperature fields in areas adjacent to the zones of coolant evaporation and condensation. Evaporation process on the boundary $\mathrm{Z}=0.015$ leads to a drastic reduction in temperature $\mathrm{T}$ of the vapor channel, adjacent to this area. The increase in heat flux to the phase transition surface leads to the certain temperature increase (as might be expected), but all the selected patterns are persist.

Figure 6 shows the effect of Reynolds number on the dimensionless heat transfer coefficient $\mathrm{Nu}$. It can be seen that increasing values of Re of 1.5 leads to a significant increase in $\mathrm{Nu}$. In general, there is an intensive heat sink from the heated surface of the thermosyphon bottom cover. Achieved values of the average Nusselt number correspond to the high thermosyphons operating efficiency. It can be concluded about the possibility of use such heat pipes to cool power equipment of thermal power plants - gas turbine blade.

According to the results of numerical analysis it was found that by using water as a coolant it is possible to achieve a certain cooling efficiency of blocks and units, heating in emergency mode to temperatures substantially exceeding permissible. But it is possible to use other refrigerants. In this case, thermosyphons operating efficiency can be even higher. Mathematical model (1)-(6) with appropriate boundary conditions can be used to analyze thermosyphons operating conditions with any cooler. For that it's enough to know liquid and vapor characteristics, and phase transition parameters.

It should be emphasized that the main result of the numerical analysis is to validate the possibility of using thermosyphons as basic elements for cooling systems of thermal power stations equipment. At the same time, thermosiphons can be used in the main thermal mode ensuring or control system, as well as a means of emergency protection from overheating units of power equipment of thermal power plants. Energy efficiency of used in each particular case heat pipes will be determined by their size, properties of used refrigerant (coolant), operating modes and (this is probably one of the most important factors), design decisions when developing a particular construction.

\section{References}

[1] Kuznetsov G.V., Al-Ani M.A., Sheremet M.A. Numerical analysis of convective heat transfer in a closed two-phase thermosyphon Journal of Engineering Thermophysics. 2011. T. 20. No. 2. 201-210

[2] Kuznetsov G.V., Sheremet M.A. New approach to the mathematical modeling of thermal regimes for electronic equipment Russian Microelectronics. 2008. T. 37. No. 2. C. 131-138

[3] Kuznetsov G.V., Sheremet M.A. Mathematical modelling of complex heat transfer in a rectangular enclosure// Thermophysics and Aeromechanics. 2009. T. 16. No. 1. C. 119-128

[4] Kuznetsov G.V., Sheremet M.A. Two-dimensional problem of natural convection in a rectangular domain with local heating and heat-conducting boundaries of finite thickness Fluid Dynamics. 2006. T. 41. No. 6. C. $881-890$

[5] Kuznetsov G.V., Sheremet M.A. Conjugate natural convection in an enclosure with local heat sources Computational Thermal Sciences. 2009. T. 1. No. 3. C. $341-360$ 